\title{
Unstable drift mode driven by shear plasma flow in solar spicules
}

\author{
H. Saleem ${ }^{1}$, J. Vranjes ${ }^{2,3}$, and S. Poedts ${ }^{2}$ \\ 1 Theoretical Plasma Physics Division, PINSTECH, PO Nilore, Islamabad, Pakistan \\ 2 Center for Plasma Astrophysics, K.U. Leuven, Celestijnenlaan 200B, 3001 Leuven, Belgium \\ e-mail: Jovo.Vranjes@wis.kuleuven.be \\ 3 Faculté des Sciences Appliquées, avenue F.D. Roosevelt 50, 1050 Bruxelles, Belgium \\ Received 3 April 2007 / Accepted 15 May 2007
}

ABSTRACT

\begin{abstract}
Context. The lower solar atmosphere contains at any moment a large number of spicules comprising plasma that moves towards the upper layers with typical axial velocities of $20-30 \mathrm{~km} \mathrm{~s}^{-1}$. It is expected that these flows as well as the plasma density are inhomogeneous in the perpendicular direction. The presence of such a density gradient implies the existence of drift waves, while the inhomogeneity of the flow velocity can cause the growth of such modes.

Aims. The stability of the drift waves will be discussed within the two-fluid theory taking into account the ion temperature and the stress tensor effects.

Methods. An analytical linear normal mode analysis is used within the local approximation.

Results. A detailed derivation of the hot ion contribution is performed. A dispersion equation is derived and the stability/instability conditions are discussed in detail for the parameter range appropriate for solar spicules. The drift mode appears to be highly unstable for typical spicule characteristic lengths of the density and the shear flow gradients, i.e. in the range of a few hundred meters up to a few kilometers, yielding wave frequencies of the order of a few $\mathrm{Hz}$

Conclusions. Hence, the waves and the instabilities develop at reasonable time scales regarding the life times of spicules that are measured in minutes.
\end{abstract}

Key words. Sun: chromosphere - waves - instabilities

\section{Introduction}

The development of the theory on drift wave has started more than 40 years ago in an attempt of explaining the anomalous diffusion of plasma across the magnetic field lines, which at that time appeared to be due to some instability of unknown origin. According to Chen (1965), the first result on that issue was due to Moiseev \& Sagdeev (1963), although already in 1961 a work has been published (Rudakov \& Sagdeev 1961) dealing with waves in inhomogeneous plasmas in the presence of both temperature and density gradients. The term "drift wave" appears to be used for the first time in the sense we use it nowadays in the work of Kadomtsev \& Timofeev (1963). We note that, in the same period, a first report on an experimental verification of lowfrequency ion waves propagating perpendicular to the magnetic field lines in the presence of a density gradient has been published by D'Angelo \& Motley (1963). Very useful books dealing with the theory of low frequency drift waves are those of Manheimer \& Lashmore-Davies (1989) and the book of Weiland (2000). In our recent works, we have been dealing with various aspects of the drift wave in both laboratory and space plasmas, see Vranjes \& Poedts (2004a,b), Vranjes \& Poedts (2005), Vranjes \& Poedts (2006a,b,c), Ali et al. (2007)

The drift wave itself is driven by a density gradient in the direction perpendicular to the magnetic field lines, and the frequency of the mode is close to the diamagnetic frequency. Such a density inhomogeneity is a very common plasma feature and its frequent presence in plasmas implies the possibility for a wide spread appearance of the drift waves. Within the kinetic theory, the mode is unstable (growing) due to the inverse electron Landau damping effect, even for a Maxwellian electron distribution; all what is needed is the presence of a density gradient which results in a mode frequency slightly below the diamagnetic frequency. On the other hand, within the fluid theory, the mode is also unstable due to the common effect of the electron collisions and the ion inertia, in the presence of the background density gradient of course. Hence, the term "universally unstable mode", used in the early literature on the drift wave. A comparison between the two instabilities (Goldston \& Rutherford 1995) reveals that the resistive one is dominant, provided that the electron parallel mean-free path is smaller than the parallel wavelength. This is essentially the reason for the interest in the drift modes with very large parallel wave-lengths and relatively short perpendicular wave-lengths. In fact, such situations occur particularly frequent in magnetized space plasmas with almost unlimited scales in the direction along the magnetic field lines.

The drift mode can be made more unstable in the presence of a plasma current or flow along the magnetic field lines, provided that this background velocity has a gradient in the perpendicular direction. A mode like the drift wave, propagating obliquely with respect to this inhomogeneous equilibrium flow, can become unstable when certain conditions are met. The first demonstration of this instability in a magnetized plasma has been given in the early work of D'Angelo in 1965. An inhomogeneous ion flow with a gradient in the $x$-direction was considered along an external magnetic field $\boldsymbol{B}_{0}=B_{0} z$. The electron and ion temperatures were equal, i.e. $T_{i}=T_{\mathrm{e}}$, and the density gradient was assumed to be oriented along the negative $x$-axis.

In the present study, assuming the same geometry, we describe the properties of the electrostatic drift wave in a hot and fully ionized plasma taking into account the ion temperature, i.e. the ion stress tensor effects. We consider a current-less case, i.e. the plasma as a whole is assumed to be flowing along the 
magnetic field lines, and we allow for the possibility that the plasma flow may be inhomogeneous in the perpendicular direction. The new results will show that the shear flow instability in such a nonuniform plasma can have a real frequency comparable to the drift wave frequency. The case of a homogeneous plasma is also modified by a small term which appears due to the collision-less stress tensor when the ion temperature has some finite value.

The origin and properties of the solar spicules have been the subject of numerous studies in the past. This is due to the fact that they have been known for more than 100 years and they are still not completely understood. In some papers their generation is attributed to the action of the nonlinear (Hollweg et al. 1982), or linear Alfvén wave like in the model of Haerendel (1992) in which their weak damping due to ion-neutral collisions results in a lift force which carries the cool, weakly ionized and dense plasma/gas mixture towards a higher altitude. Yet, in the same work, the lack of a permanent source for such Alfvén waves is explicitly pointed out. In some models (see the review work Narain \& Ulmschneider 1990, and references cited therein, and also Priest 1987) they appear by sucking of the plasma by nearly evacuated magnetic flux tubes and by a consequent supersonic expansion of the material in the vertical direction. More details on the various models are available in the review of Sterling (2000). In more recent works (De Pontieu et al. 2004; De Pontieu \& Erdélyi 2006) the observed quasi-periodicity of spicules (around $5 \mathrm{~min}$, reported first by Kulidzanishvili \& Nikolsky 1978; Kulidzanishvili \& Zhugzhda 1983), has been discussed in relation with the solar surface pressure (p) modes. Like in the spicules that appear as skeletal structures in sponges, this model assumes that the increase of pressure (caused by the p-modes) in the lower layers, results in the focusing of the plasma in the magnetic flux tubes. As a result, there should be a lifting of the plasma along the inclined tubes and a resulting horizontal density stratification resembling a grassy structure, as seen on the solar limb. The origin of spicules is out of the scope of the present work. Rather, we here focus on some features of spicules, i.e., on the existence of the plasma flow and its possible role in the excitation of electrostatic, growing drift waves. In Sect. 2 we derive the dispersion equation for the drift wave in plasmas with hot ions, and in Sect. 3 we apply the results to the solar spicules. At the end, a Summary is presented.

\section{Model and derivations}

Let us consider an inhomogeneous magnetized plasma embedded in an external homogeneous magnetic field $\boldsymbol{B}_{0}=B_{0} \boldsymbol{z}=$ const., with a parallel shear plasma flow defined as $\boldsymbol{v}_{j 0}=$ $v_{j 0}(x) z$, and a density inhomogeneity that is defined as $\nabla n_{j 0}=$ $-\boldsymbol{x} \mathrm{d} n_{j 0} / \mathrm{d} x$. Here, the subscript $j=\mathrm{e}, \mathrm{i}$ denotes electrons and ions, respectively, having temperatures $T_{j}$ and masses $m_{j}$. The parallel flow is driven externally. The steady state demands that each species has a zero-order diamagnetic drift $\boldsymbol{v}_{\perp j 0}=\boldsymbol{v}_{D j 0}=$ $-\left(T_{j} / q_{j} B_{0}\right) \nabla \ln n_{j 0} \times z$, where $q_{j}$ is the charge on $j$ th species.

For electrostatic perturbations, the perpendicular component of the perturbed velocity can be expressed by the following recurrent formula,

$\boldsymbol{v}_{\perp j}=\frac{1}{B_{0}}\left(\boldsymbol{E}_{\perp} \times z\right)-\frac{T_{j}}{q_{j} B_{0}}\left(\nabla \ln n_{j} \times z\right)-\frac{1}{q_{j} B_{0}}\left(\frac{\nabla \cdot \boldsymbol{\Pi}_{j}}{n_{j}}\right)$

$-\frac{1}{\Omega_{j}}\left(\partial_{t}+v_{j} \cdot \nabla\right) v_{j} \times z=v_{\mathrm{E}}+v_{\mathrm{D} j}+\boldsymbol{v}_{\Pi j}+v_{\mathrm{p} j}$
Here, $\boldsymbol{v}_{\mathrm{E}}, \boldsymbol{v}_{\mathrm{D} j}, \boldsymbol{v}_{\Pi j}$ and $\boldsymbol{v}_{\mathrm{p} j}$ are the electric, diamagnetic, stress tensor and polarization drifts of the $j$ th species, respectively, and $\Omega_{j}=q_{j} B_{0} / m_{j}$ is the gyro frequency.

Since $\nabla \cdot \boldsymbol{v}_{\mathrm{E}}=0$, the continuity equation reduces to,

$\partial_{t} n_{j}+\nabla n_{j 0} \cdot \boldsymbol{v}_{\mathrm{E}}+\nabla_{\perp} \cdot\left[n_{j}\left(\boldsymbol{v}_{\mathrm{p} j}+\boldsymbol{v}_{\Pi j}\right)\right]+\partial_{z}\left(n_{j} v_{j z}\right)=0$.

In the linear limit we have,

$\nabla \cdot\left[n_{j}\left(\boldsymbol{v}_{\mathrm{p} j}+\boldsymbol{v}_{\Pi j}\right)\right]_{1}=\nabla \cdot\left[\frac{n_{j 0}}{\Omega_{j}} \partial_{t}\left(z \times \boldsymbol{v}_{j 1}\right)\right]$,

which is the same as Eq. (2.41) of Weiland (2000). If $\boldsymbol{v}_{j 1}=\boldsymbol{v}_{\mathrm{E} 1}+$ $v_{\mathrm{D} j 1}$ is assumed for drift waves, then Eq. (3) becomes

$$
\begin{gathered}
\nabla \cdot\left[n_{j}\left(\boldsymbol{v}_{\mathrm{p} j}+\boldsymbol{v}_{\Pi j}\right)\right]_{1}=\frac{n_{j 0}}{B_{0} \Omega_{j}} \partial_{t}\left(\nabla_{\perp} \cdot \boldsymbol{E}_{\perp 1}\right)-\frac{T_{j}}{q_{j} B_{0} \Omega_{j}} \partial_{t} \nabla_{\perp}^{2} n_{j 1} \\
+\frac{n_{j 0}}{\Omega_{j}} v_{j z 0}(x) \partial_{z}\left[\frac{1}{B_{0}} \nabla_{\perp} \cdot \boldsymbol{E}_{\perp 1}-\frac{T_{j}}{q_{j} B_{0}} \frac{\nabla_{\perp}^{2} n_{j 1}}{n_{j 0}}\right] .
\end{gathered}
$$

Equations (2) and (4) yield

$$
\begin{aligned}
& \left(\partial_{t}+v_{j z 0} \partial_{z}\right) n_{j 1}+\nabla n_{j 0} \cdot \boldsymbol{v}_{\mathrm{E} 1}+\frac{n_{j 0}}{B_{0} \Omega_{j}}\left(\partial_{t}+v_{j z 0} \partial_{z}\right)\left(\nabla_{\perp} \cdot \boldsymbol{E}_{\perp 1}\right) \\
& -\frac{T_{j}}{q_{j} B_{0} \Omega_{j}}\left(\partial_{t}+\boldsymbol{v}_{j} \cdot \nabla\right) \nabla_{\perp}^{2} n_{j 1}+n_{j 0} \partial_{z} v_{j z 1}=0 .
\end{aligned}
$$

Using the relation

$-\mathrm{i} k_{z} c_{\mathrm{i}}^{2} \rho_{\mathrm{i}}^{2} k_{y}^{2}\left(\frac{e \varphi_{1}}{T_{\mathrm{i}}}+\frac{n_{\mathrm{i} 1}}{n_{\mathrm{i} 0}}\right)+\left(\boldsymbol{v}_{D \mathrm{i} 0} \cdot \nabla\right) v_{\mathrm{i} z 1}=-\frac{1}{m_{\mathrm{i}}}\left(\frac{\nabla \cdot \boldsymbol{\Pi}_{\mathrm{i}}}{n_{\mathrm{i}}}\right)_{1}$,

the parallel component of the ion equation of motion can be written in the linear limit as

$\left(\partial_{t}+v_{\mathrm{i} z 0} \partial_{z}\right) v_{\mathrm{i} z 1}+v_{\mathrm{i} x 1} \partial_{x} v_{\mathrm{i} z 0}(x)=\frac{q_{j}}{m_{\mathrm{i}}} E_{z 1}-\frac{T_{\mathrm{i}}}{m_{\mathrm{i}} n_{\mathrm{i} 0}} \partial_{z} n_{\mathrm{i} 1}$

$-\mathrm{i} k_{z} c_{\mathrm{i}}^{2} \rho_{\mathrm{i}}^{2} k_{y}^{2}\left(\frac{e \varphi_{1}}{T_{\mathrm{i}}}+\frac{n_{\mathrm{i} 1}}{n_{\mathrm{i} 0}}\right)$

Here $\boldsymbol{E}_{1}=-\nabla \varphi_{1}$. For hot ions, Eqs. (5) and (7) become, respectively,

$\Omega_{\omega}^{2} \frac{n_{\mathrm{i} 1}}{n_{\mathrm{i} 0}}-\omega_{\mathrm{e}}^{*} \Omega_{\omega} \Phi_{1}+\left(\rho_{\mathrm{s}}^{2} k_{y}^{2} \Phi_{1}+\rho_{\mathrm{i}}^{2} k_{y}^{2} \frac{n_{\mathrm{i} 1}}{n_{\mathrm{i} 0}}\right) \Omega_{\omega}-k_{z} v_{\mathrm{i} z 1}=0$,

and

$\Omega_{\omega} v_{\mathrm{i} z 1}+\left(\frac{1}{\Omega_{\mathrm{i}}} \partial_{x} v_{\mathrm{i} z 0}\right) c_{\mathrm{s}}^{2} k_{y} \Phi_{1}=\left(1+\rho_{\mathrm{i}}^{2} k_{y}^{2}\right)\left(c_{\mathrm{s}}^{2} k_{z} \Phi_{1}+c_{\mathrm{i}}^{2} k_{z} \frac{n_{\mathrm{i} 1}}{n_{\mathrm{i} 0}}\right)$

Here,

$\Omega_{\omega}=\omega-\omega_{z 0}, \quad \omega_{z 0}=v_{\mathrm{i} z 0} k_{z}, \quad \omega_{\mathrm{e}}^{*}=\boldsymbol{k} \cdot \boldsymbol{v}_{\mathrm{De} 0}=k_{y} T_{\mathrm{e}} \kappa_{n \mathrm{e}} / e B_{0}$,

$\kappa_{n \mathrm{e}}=\mathrm{d} n_{\mathrm{e} 0} /\left(n_{\mathrm{e} 0} \mathrm{~d} x\right), \quad \rho_{\mathrm{s}}^{2}=c_{\mathrm{s}}^{2} / \Omega_{\mathrm{i}}^{2}, \quad c_{\mathrm{s}}^{2}=T_{\mathrm{e}} / m_{\mathrm{i}}$,

$c_{\mathrm{i}}^{2}=T_{\mathrm{i}} / m_{\mathrm{i}}, \quad \rho_{\mathrm{i}}^{2}=c_{\mathrm{i}}^{2} / \Omega_{\mathrm{i}}^{2}, \quad \Phi_{1}=e \varphi_{1} / T_{\mathrm{e}}$.

For the $v_{j 0}=0$ case, we have approximately $n_{\mathrm{i} 1} / n_{\mathrm{i} 0} \simeq n_{\mathrm{e} 1} / n_{\mathrm{e} 0} \simeq$ $\omega_{\mathrm{e}}^{*} \Phi_{1}$, so that

$\nabla \cdot\left[n_{\mathrm{i}}\left(\boldsymbol{v}_{\mathrm{pi}}+\boldsymbol{v}_{\Pi \mathrm{i}}\right)\right]_{1} \simeq-\mathrm{i} n_{\mathrm{i} 0} k_{y}^{2} \rho_{\mathrm{s}}^{2}\left(\omega-\omega_{\mathrm{i}}^{*}\right) \Phi_{1}$, 
which is the same as Eq. (2.43) of Weiland (2000). The electromagnetic dispersion relation (4.47) of Weiland (2000) then reduces to the electrostatic limit for $c_{\mathrm{s}}^{2} \ll v_{\mathrm{A}}^{2}$ (where $v_{\mathrm{A}}$ is the Alfvén speed) as,

$\omega^{2}-\omega_{\mathrm{e}}^{*} \omega-c_{\mathrm{s}}^{2} k_{z}^{2}=-\rho_{\mathrm{s}}^{2} k_{y}^{2}\left(\omega-\omega_{\mathrm{i}}^{*}\right) \omega$.

For the present case of the two-component electron-ion plasma assuming

$n_{1 \mathrm{e}} \simeq n_{\mathrm{e} 0} \mathrm{e}^{\mathrm{e} \varphi_{1} / T_{\mathrm{e}}} \simeq n_{\mathrm{e} 0} \frac{\mathrm{e} \varphi_{1}}{T_{\mathrm{e}}}$,

and with the help of the Poisson equation we have

$$
\begin{array}{r}
\left(1+\rho_{\mathrm{s}}^{2} k_{y}^{2}+\rho_{\mathrm{i}}^{2} k_{y}^{2}\right) \Omega_{\omega}^{2}-\omega_{\mathrm{e}}^{*} \Omega_{\omega}+A_{\mathrm{i}} c_{\mathrm{s}}^{2} k_{y} k_{z} \\
-\left(1+\rho_{\mathrm{i}}^{2} k_{y}^{2}\right)\left(c_{\mathrm{s}}^{2}+c_{\mathrm{i}}^{2}\right) k_{z}^{2}=0 .
\end{array}
$$

Here, $\Gamma=\mathrm{d} v_{z 0} /\left(\Omega_{\mathrm{i}} \mathrm{d} x\right)$ is the dimensionless shear. In the case of $v_{z 0}=0$, and using $n_{\mathrm{i} 1} / n_{\mathrm{i} 0} \sim \omega_{\mathrm{e}}^{*} \Phi_{1}$, Eq. (13) reduces to Eq. (11).

For $T_{\mathrm{e}}=T_{\mathrm{i}}=T$, the dispersion relation (13) becomes

$\left(1+2 \rho_{\mathrm{i}}^{2} k_{y}^{2}\right) \Omega_{\omega}^{2}-\omega_{\mathrm{e}}^{*} \Omega_{\omega}+\Gamma c_{\mathrm{i}}^{2} k_{z} k_{y}-\left(1+\rho_{\mathrm{i}}^{2} k_{y}^{2}\right) 2 c_{\mathrm{i}}^{2} k_{z}^{2}=0$.

Note that even in the homogeneous limit the instability criterion of D'Angelo (1965) is modified due to the collision-less part of the ion stress tensor.

If $T_{\mathrm{i}} \ll T_{\mathrm{e}}$ is assumed in Eq. (14), we obtain

$\left(1+\rho_{\mathrm{s}}^{2} k_{y}^{2}\right) \Omega_{\omega}^{2}-\omega_{\mathrm{e}}^{*} \Omega_{\omega}+\Gamma c_{\mathrm{s}}^{2} k_{y} k_{z}-c_{\mathrm{s}}^{2} k_{z}^{2}=0$,

which yields

$\omega=k_{z} v_{z}+\frac{1}{2 a}\left\{\omega_{\mathrm{e}}^{*} \pm\left[\omega_{\mathrm{e}}^{* 2}-4 a\left(\Gamma c_{\mathrm{s}}^{2} k_{y} k_{z}-c_{\mathrm{s}}^{2} k_{z}^{2}\right)\right]^{\frac{1}{2}}\right\}$.

Here, $a=1+\rho_{\mathrm{s}}^{2} k_{y}^{2}$. From Eq. (13) the instability sets in provided that

$\Gamma>f\left(k_{y}, k_{z}, L_{n}\right)=2\left(1+\rho_{\mathrm{i}}^{2} k_{y}^{2}\right) \frac{k_{z}}{k_{y}}+\frac{1}{4\left(1+2 \rho_{\mathrm{i}}^{2} k_{y}^{2}\right)} \frac{\rho_{\mathrm{i}}^{2}}{L_{n}^{2}} \frac{k_{y}}{k_{z}}$.

The minimum of $f$ in the $\left(k_{y}, k_{z}\right)$ plane is obtained for

$k_{z}=\frac{1}{8\left(1+\rho_{i}^{2} k_{y}^{2}\right)\left(1+2 \rho_{i}^{2} k_{y}^{2}\right)} \frac{\rho_{i}^{2}}{L_{n}^{2}}$.

In the case of a homogeneous plasma and for cold ions, our result is the same as that of D'Angelo (1965), and the real part of the frequency is $\omega_{r}=k_{z} v_{z 0}$.

\section{Application to solar spicules}

From the physical point of view the instability discussed above is undisputable and it has even been confirmed experimentally, see e.g. D'Angelo \& Goeler (1965) and Luo et al. (2001). Yet, the formal conditions obtained in the frame of the local approximation may be far from reality and a nonlocal approach would become more appropriate. This implies solving an appropriate differential equation for the spatially varying mode amplitude. An analysis of that kind has been performed by Basu \& Coppi (1989), but for a different plasma and for different modes, and the results have been compared with those obtained from the local approach, showing a reasonable agreement.

One example where our local analysis and the condition (17) seem to be fairly well applicable is the instability of the drift

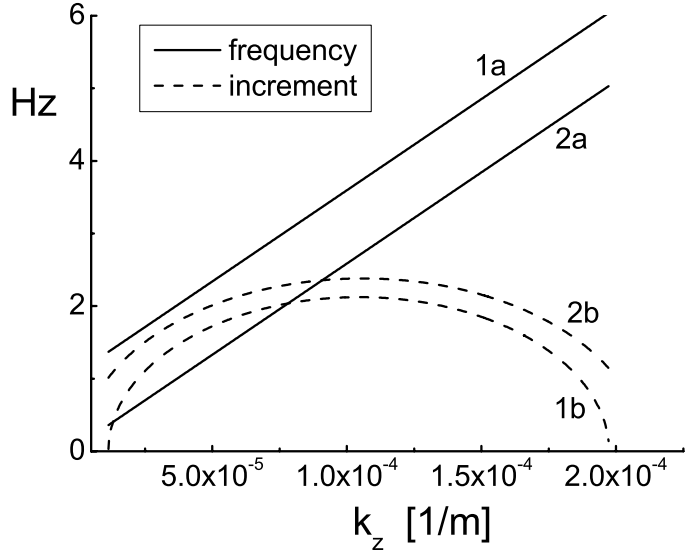

Fig. 1. The frequencies and the increments of the shear-flow-driven drift mode in terms of $k_{z}$ for $\lambda_{y}=5 \mathrm{~m}$, and for $L_{n}=100 \mathrm{~m}$ (lines 1) and $L_{n}=1 \mathrm{~km}$ (lines 2).

wave in the solar spicules. Here, we have Priest (1987) plasma jets with typical velocities in the range of about 20 to $30 \mathrm{~km} \mathrm{~s}^{-1}$, usually ejected along the magnetic field lines. The velocity is known to remain constant over long distances and the spicules have typical life time about 5 to $10 \mathrm{~min}$. The density is typically in the range $10^{16}-10^{17} \mathrm{~m}^{-3}$ and we adopt the temperature $T_{\mathrm{e}} \sim T_{\mathrm{i}}=2 \times 10^{4}$. In view of the limited life-time of the solar spicules, here we are interested in relatively high frequency modes, i.e., in relatively short density-scale gradients. For the electron-proton plasma applicable to the case, we take the following set of parameters: $B_{0}=10^{-2} \mathrm{~T}, v_{z 0}=20 \mathrm{~km} \mathrm{~s}^{-1}$, $L_{n}=L_{v}=100 \mathrm{~m}, k_{z} / k_{y}=10^{-4}$, more specifically we choose $\lambda_{z}=50 \mathrm{~km}$ and $\lambda_{y}=5 \mathrm{~m}$. For such parameter values, the assumptions used in the derivations (i.e. the local analysis, negligible electron inertia) are well satisfied. The plasma $\beta$ is about $7 \times 10^{-5}$, justifying the electrostatic limit used in the analysis. The diamagnetic frequency becomes $\omega_{\mathrm{e}}^{*}=2.16 \mathrm{~Hz}$, while $k_{z} v_{z 0}=2.5 \mathrm{~Hz}$, and the wave frequency becomes $\omega \simeq 4+\mathrm{i} 1.3 \mathrm{~Hz}$. The present set of parameters is chosen arbitrarily and shows a strongly growing shear-flow-driven drift mode. The inhomogeneity scales used here are expected to be present in the outer region of a plasma jet which is directed along the magnetic lines in the spicules. For the set of parameters given above, and for the given fixed $k_{y}$, we vary $k_{z}$ in a range of values. The result is presented in Fig. 1 showing the instability window for the flow driven mode. The curves shown in Fig. 1 are for two values of the density gradient length, viz. $L_{n}=10^{2}$ and $10^{3} \mathrm{~m}$ (lines $a$ and $b$, respectively). A larger $L_{n}$ implies a lower frequency of the drift mode and a higher increment.

A similar mode behavior is observed for a fixed parallel wave-number $k_{z}=1.2 \times 10^{-4} \mathrm{~m}^{-1}$ and for the perpendicular wave-number $k_{y}$ taking values in the interval 0.64 and 11.3 , i.e., for the perpendicular wave-lengths of about $0.5 \mathrm{~m}$ to $10 \mathrm{~m}$. The result is given in Fig. 2. Note the strong growing of the mode for the weaker density gradient $\left(L_{n}=1 \mathrm{~km}\right)$.

The mode is examined also by changing the shear length $L_{v}$. This is presented in Fig. 3. In accordance with the dispersion equation, the real frequency is constant up to the threshold value of the shear length which is here around $1700 \mathrm{~m}$. The other parameters are as follows: $L_{n}=1 \mathrm{~km}, \lambda_{z}=200 \mathrm{~km}, \lambda_{y}=2 \mathrm{~m}$. The magnetic field and the temperature is kept the same as in the previous graphs.

The threshold (17) is presented in Fig. 4 for the fixed value $\lambda_{z}=50 \mathrm{~km}$, showing a remarkable dip along the $k_{y}$-axis, i.e., an 


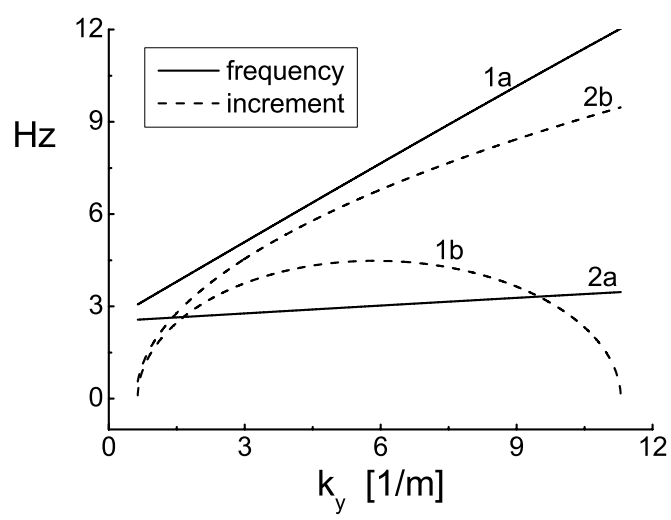

Fig. 2. The frequencies and the increments in terms of $k_{y}$ for a fixed $k_{z}=1.2 \times 10^{-4} \mathrm{~m}^{-1}$ for $L_{n}=100 \mathrm{~m}$ (lines 1) and $L_{n}=1 \mathrm{~km}$ (lines 2).

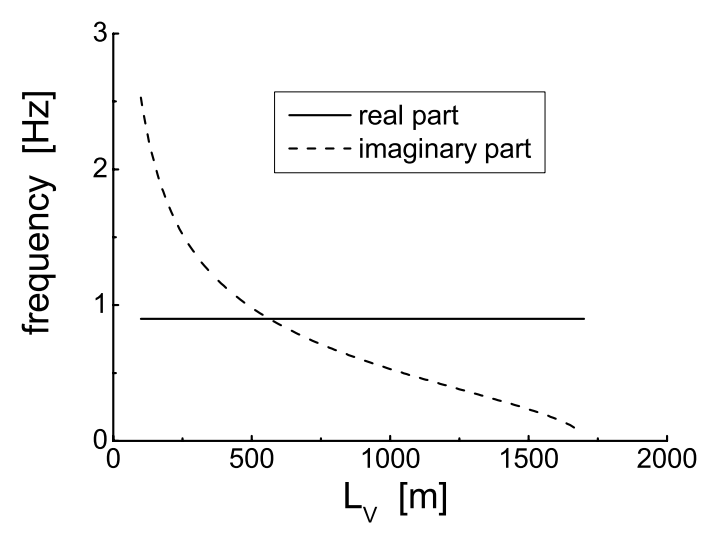

Fig. 3. The frequency and the increment of the drift mode versus the shear flow inhomogeneity length $L_{v}$, for the parameters given in the text.

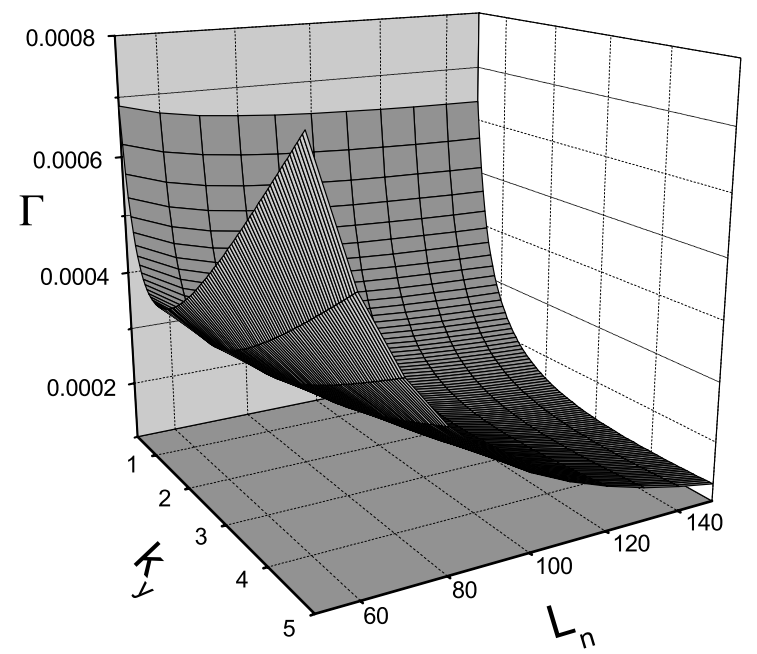

Fig. 4. The minimal value of the normalized shear $\Gamma$ in terms of $k_{y}$ and $L_{n}$. Instability sets in for the values above the surface.

angle dependant threshold, and a decrease in the threshold for a weaker inhomogeneity which is most evident in the domain of large values of $k_{y}$, both of these effects being in agreement with Fig. 2. The corresponding contour plot in Fig. 5 features the same effects.

In the Cartesian geometry and the local analysis used here, the mode (13) implies an electrostatic wave with perpendicular

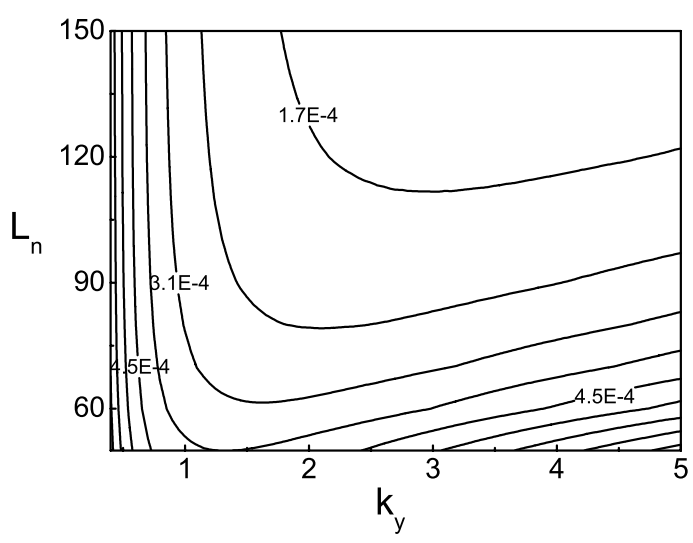

Fig. 5. The contour plot of the normalized shear $\Gamma$.

and parallel potential/density perturbations. In a realistic model geometry of the solar spicules, the signatures of such waves should be sought in the form of twisted structures, highly elongated along the axis of the spicules and traveling along the axis with the speed close to the local sound velocity (this of course in the reference frame moving with the velocity $v_{z 0}$ ). Solving the starting equations in such a geometry would involve differential equations with the corresponding eigenfunctions in terms of the Kummer (Vranjes \& Poedts 2004a) or the Bessel functions (Vranjes \& Poedts 2004b).

\section{Summary}

Presently, there seems to be no definite consensus about the origin of the spicules in the lower solar atmosphere but numerous attempts have been made to explain their origin and their possible role in the heating of the upper layers. Yet, it is widely accepted that the total mass flux carried upwards by spicules is much larger than the global solar wind flux and, therefore, these jets of relatively cool matter are followed by a downflow of plasma with a one order of magnitude higher temperature. Hence, somewhere at higher altitudes some heating takes place, although it is still not well understood what really happens once when spicules vanish from the visual spectrum. Regarding the origin of solar spicules, we would like to point to a recent work (Chen 2006) dealing with the sheath theory and the double layer formation in the free space, i.e. away from boundaries. According to this result, a current-less plasma acceleration appears in the geometry of diverging magnetic lines (typically the case in the solar atmosphere) with the only assumption of Maxwellian electrons.

The spicules extend high enough to play a role in the heating of the lower corona, and for that purpose the excitation of waves which would in the end inevitably dissipate may be proposed. Simple estimates show that, if the macroscopic flow energy per unit volume of relatively dense plasma in spicules would convert into a thermal energy of the rarified plasma in the lower corona, this would result in a raise of the temperature of at least 2 orders of magnitude. The excitation of the modes discussed in the present work should be understood in this context. The dispersion Eq. (14) describes the oscillatory instability of the electrostatic drift wave due to the presence of the shear in the plasma flow. The mode is shown to be strongly growing for the realistic parameter values used here. The postulated density inhomogeneity and the shear in the plasma flow should be expected as facts in solar spicules. A drift wave excited by the mechanism presented above, in its propagation through the plasma can be efficiently 
dissipated in the regions with inhomogeneous (sheared) magnetic field, and in such a way contribute to the plasma heating. An interesting feature of this effect is that the wave dissipates directly on ions. In the sheared magnetic field (i.e., spatially varying parallel wave number $\left.k_{\|}\right)$this appears as a Landau dissipation in the regions where $k_{\|} c_{\mathrm{i}}=\omega$ (Weiland 2000).

The fact that the frequency of the mode is the drift wave frequency (see Eq. (16)), has been overlooked in the past in the studies dealing with the shear flow instability in spatially inhomogeneous plasmas. The same holds for the ion temperature effects. Namely, in the calculation of the ion terms $\nabla\left(n_{\mathrm{i}} v_{\mathrm{i}}\right)$, it turns out that the contribution of the ion diamagnetic drift to the convective derivative in the polarization drift is in fact canceled out by a part of the stress tensor, as demonstrated in this work by Eq. (3).

We note that for the given parameters, the collision frequencies, although omitted in the derivations, are much higher than the wave frequencies, so the fluid model used in this work is appropriate. In the same time, the kinetic effects are of no importance, for both electrons and ions. Using $v_{\mathrm{ei}}=\left(8 \pi / m_{\mathrm{e}}\right)^{1 / 2}\left[e^{2} /\left(4 \pi \varepsilon_{0}\right)\right]^{2} n_{0} \Lambda /(\kappa T)^{3 / 2}$, where $\Lambda=\ln \left[12 \pi \varepsilon_{0}\left(\varepsilon_{0} / n_{0}\right)^{1 / 2}(\kappa T)^{3 / 2} / e^{3}\right]$, we obtain for electrons $v_{\mathrm{ei}}=82 \times 10^{4} \mathrm{~Hz}$. Similarly, for ions we have $v_{\mathrm{ei}}=$ $4\left(\pi / m_{\mathrm{i}}\right)^{1 / 2}\left[e^{2} /\left(4 \pi \varepsilon_{0}\right)\right]^{2} n_{0} \Lambda /\left[3(\kappa T)^{3 / 2}\right]=2.7 \times 10^{3} \mathrm{~Hz}$. For such a relatively short ion collision time, in comparison to the wave period, the Landau damping on hot ions is not expected to play any essential role. In the same time, in view of the discussion given in the Introduction and due to the fact that the electron mean free path is several orders of magnitude shorter than the parallel wave-length, the electron dissipative amplification of the drift wave will additionally destabilize the mode. These dissipative effects are discussed in more detail by Vranjes \& Poedts (2006c).
Acknowledgements. These results are obtained in the framework of the projects G.0304.07 (FWO-Vlaanderen), C 90203 (Prodex), GOA/2004/01 (K.U.Leuven), and the Interuniversity Attraction Poles Programme - Belgian State - Belgian Science Policy.

\section{References}

Ali, A., Saleem, H., Vranjes, J., \& Poedts, S. 2007, Phys. Lett. A, 366, 466 Basu, B., \& Coppi, B. 1989, J. Geophys. Res. A, 94, 5316

Chen, F. F. 1965, Phys. Fluids, 8, 1323

Chen, F. F. 2006, Phys. Plasmas, 13, 034502

D'Angelo, N. 1965, Phys. Fluids, 8, 1748

D’Angelo, N., \& Motley, R.V. 1963, Phys. Fluids, 6, 422

D'Angelo, N., \& Goeler, S. V. 1965, Phys. Fluids, 9, 309

De Pontieu, B., Erdélyi, R., \& James, S. P. 2004, Nature, 430, 536

De Pontieu, B., \& Erdélyi, R. 2006, Philos. Trans. Roy. Soc. A, 364, 383

Goldston, R. J., \& Rutherford, P. H. 1995, Introduction to Plasma Physics (Institute of Physics Pub., Bristol and Philadelphia)

Haerendel, G. 1992, Nature, 360, 241

Hollweg, J. V., Jackson, S., \& Galloway, D. 1982, Sol. Phys., 75, 35

Kadomtsev, B. B., \& Timofeev, A.V. 1963, Sov. Phys. Doklady, 7, 826

Kulidzanishvili, V. I., \& Nikolsky, G. M. 1978, Sol. Phys., 59, 21

Kulidzanishvili, V. I. \& Zhugzhda, Y. D. 1983, Sol. Phys., 88, 35

Luo, Q. Z., D’Angelo, N., \& Merlino, R. L. 2001, Phys. Plasmas, 8, 31

Manheimer, W. M., \& Lashmore-Davies, C. N. 1989, MHD and Microinstabilities in Confined Plasma (Institute of Physics Pub., Bristol)

Moiseev, S. S., \& Sagdeev, R. Z. 1963, J. Exp. Theor. Phys. (U.S.S.R.) 44, 763

Narain, U., \& Ulmshneider, P. 1990, Space Sci. Rev., 54, 377

Priest, E. R. 1987, Solar Magnetohydrodynamics (D. Reidel Pub. Comp., Dordrecht)

Rudakov, L. I., \& Sagdeev, R. Z. 1961, Sov. Phys. Doklady, 6, 415

Sterling, A. C. 2000, Sol. Phys., 196, 79

Vranjes, J., \& Poedts, S. 2004a, Phys. Plasmas, 11, 891

Vranjes, J., \& Poedts, S. 2004b, Phys. Plasmas, 11, 2178

Vranjes, J., \& Poedts, S. 2005, Phys. Plasmas, 12, 064501

Vranjes, J., \& Poedts, S. 2006a, Phys. Lett. A, 348, 346

Vranjes, J., \& Poedts, S. 2006b, Phys. Plasmas, 13, 032107

Vranjes, J., \& Poedts, S. 2006c, A\&A, 458, 635

Weiland, J. 2000, Collective Modes in Inhomogeneous Plasmas (Bristol: Institute of Physics Pub.) 